\title{
Double-blind maintenance safety and effectiveness findings from the Treatment of Early-Onset Schizophrenia Spectrum Study (TEOSS)
}

\author{
Robert L Findling, MD, Jacqueline L Johnson, DrPH, Jon McClellan, MD, Jean A Frazier, \\ MD, Benedetto Vitiello, MD, Robert M Hamer, PhD, Jeffrey A Lieberman, MD, Louise Ritz, \\ MBA, Nora K McNamara, MD, Jacqui Lingler, BS, Stefanie Hlastala, PhD, Leslie Pierson, \\ MPH, Madeline Puglia, BA, Ann E Maloney, MD, Emily Michael Kaufman, MA, Nancy Noyes, \\ PMH-NP, and Linmarie Sikich, MD \\ Drs. Findling and McNamara and Ms. Lingler are with the Department of Psychiatry, Case Western \\ Reserve University. Drs. McClellan and Hlastala are with the University of Washington. Ms. Pierson \\ is with the Division of Child Psychiatry, Seattle Children's Hospital. Dr. Frazier is with UMASS \\ Medical School. Ms. Michael Kaufman is with Massachusetts School of Professional Psychology. \\ Dr. Vitiello is with National Institute of Mental Health. Ms. Ritz is with the National Institute of \\ Neurological Disorders and Stroke. Dr. Maloney and Ms. Noyes are with the Maine Medical Center \\ Research Institute. Dr. Lieberman is with Columbia University. Drs. Johnson, Hamer, and Sikich \\ and Ms. Puglia are with the University of North Carolina at Chapel Hill. Dr. Frazier, Ms. Michael \\ Kaufman, and Ms. Noyes were affiliated with Cambridge Health Alliance, Harvard Medical School \\ at the time when the date from the study was collected. Drs. Maloney and Lieberman were at the \\ University of North Carolina at Chapel Hill during the study.
}

\section{Abstract}

\footnotetext{
(C) 2010 American Academy of Child \& Adolescent Psychiatry. Published by Elsevier Inc. All rights reserved.

Correspondence to: Robert L Findling, MD, robert.findling @ uhhospitals.org, 10524 Euclid Avenue Suite 1155A, Cleveland OH 44106, Phone: 216-844-1717, Fax: 216-844-5883.
}

Publisher's Disclaimer: This is a PDF file of an unedited manuscript that has been accepted for publication. As a service to our customers we are providing this early version of the manuscript. The manuscript will undergo copyediting, typesetting, and review of the resulting proof before it is published in its final citable form. Please note that during the production process errors may be discovered which could affect the content, and all legal disclaimers that apply to the journal pertain.

Disclosure: Dr. Findling receives or has received research support, served as a consultant for and/or served on the speakers' bureau for Abbott, Addrenex, AstraZeneca, Biovail, Bristol-Myers Squibb, Forest, GlaxoSmithKline, Johnson and Johnson, KemPharm, Eli Lilly and Company, Lundbeck, Neuropharm, Novartis, Organon, Otsuka, Pfizer, Sanofi-Aventis, Sepracore, Shire, Solvay, Supernus Pharmaceuticals, Validus, and Wyeth. Dr. Frazier receives research funding from or participates in clinical trials with Bristol-Myers Squibb, GlaxoSmithKline, Janssen, Johnson and Johnson, Neuropharm, Otsuka America Pharmaceutical, and Pfizer. Dr. Hamer, in the past three years, received or has receives research support, served as a consultant for and served on a data safety monitoring board (DSMB) / internal displacement monitoring centre (IDMC) for Acadia, Allergan, Alpharma, AstraZeneca, Cenerx, Corcept, EnabledMD, Epix, Johnson and Johnson, Novartis, Pepper-Hamilton, Pfizer, SAS Institute, Schwartz, Solvay, Sanofi-Aventis, Takeda, WinstonStrawn (a lawsuit involving Forest, Lundbeck, Sun, Caraco), and Wyeth. He and/or his wife own stock in Bristol-Myers Squibb, Amgen, Eli Lilly and Company, Genentech, Proctor and Gamble, and Sepracor. His wife is retired from Bristol-Myers Squibb. Dr. Liberman serves on the advisory board of Bioline, GlaxoSmithKline, Intracellular Therapies, Eli Lilly and Company, Psychogenics, and Wyeth. $\mathrm{He}$ does not receive financial compensation or salary support for his participating as an advisor, except for Intracellular Therapies. He receives grant support from Allon, Forest Labs, Merck, and Pfizer. He holds a patent from Repligen.

Dr. Sikick receives research funding or participates in clinical trials with Janssen, Pfizer, Bristol-Myers Squibb, Neuropharm, Curemark, and Seaside Pharmaceuticals. She has receives software for a computer intervention in schizophrenia from Posit Science. In the past, Dr. Sikich received research funding from Eli Lilly and Company, Janssen, Pfizer, Otsuka, and AstraZeneca. She has served as a consultant for Sanofi Aventis and ABT Associates. Drs. Johnson, McClellan, Vitiello, McNamara, Hlastala, and Maloney, and Ms. Ritz, Ms. Lingler, Ms. Pierson, Ms. Puglia, Ms. Michael Kaufman, and Ms. Noyes report no biomedical financial interests or potential conflicts of interest.

Clinical trial registry information - Treatment of Schizophrenia and Related Disorders in Children and Adolescents; http://www.clinicaltrials.gov; NCT00053703 
OBJECTIVE-To examine the long-term safety and efficacy of three antipsychotics in early-onset schizophrenia spectrum disorders (EOSS).

METHOD—Patients (age 8-19 years) who had improved during an 8-week, randomized doubleblind acute trial of olanzapine, risperidone, or molindone (plus benztropine) were eligible to continue on the same medication for up to 44 additional weeks under double-blind conditions. Adjunctive medications were allowed following defined algorithms. Standardized symptom, safety, and functional assessments were conducted every 4 weeks.

RESULTS-Of the 116 youth randomized in the acute trial, 54 entered maintenance treatment (molindone, $\mathrm{N}=20$; olanzapine, $\mathrm{N}=13$; risperidone, $\mathrm{N}=21$ ). Fourteen (26\%) completed 44 weeks of treatment. Adverse effects $(\mathrm{N}=15)$, inadequate efficacy $(\mathrm{N}=14)$, or study non-adherence $(\mathrm{N}=8)$ were the most common reasons for discontinuation. The three treatment arms did not significantly differ in symptom reduction or time to discontinuation. Akathisia was more common with molindone and elevated prolactin concentrations more common with risperidone. Although weight gain and metabolic adverse events had occurred more often with olanzapine and risperidone during the acute trial, no significant between-drug differences emerged in most of these parameters during maintenance treatment.

CONCLUSIONS-Only $12 \%$ of youth with EOSS continued on their originally randomized treatment at 52 weeks. No agent demonstrated superior efficacy, and all were associated with side effects, including weight gain. Improved treatments are needed for EOSS.

\section{Keywords}

adolescent; schizophrenia; schizoaffective disorder; antipsychotic; treatment

\section{INTRODUCTION}

Early-onset schizophrenia spectrum disorders (EOSS; onset $<18$ years) are associated with disabling symptomatology, marked functional impairments, and a poor prognosis. ${ }^{1}, 2$ Safe and effective treatments are needed to improve the outcomes of these vulnerable youths.

In the largest publicly funded trial conducted to date, our Treatment of Early-Onset Schizophrenia Spectrum Disorders (TEOSS) study compared the acute effectiveness and safety of antipsychotics in youth with EOSS. Overall, approximately $50 \%$ of subjects responded to the initially randomized treatment. ${ }^{3}$ Two "second generation" antipsychotics, risperidone and olanzapine, were not superior to a "first generation" antipsychotic, molindone, in symptom diminution or proportion of treatment responders. Both risperidone and olanzapine were associated with greater degrees of weight gain than molindone. Molindone was associated with higher rates of akathisia. Olanzapine treatment also led to significant increases in fasting cholesterol, low density lipoprotein, insulin, and liver transaminases. Given these risks, the study's Data and Safety Monitoring Board discontinued further randomizations into the olanzapine arm midway through the study and required implementation of more rigorous procedures to assess the benefit/risk ratio in all participants.

As schizophrenia is a chronic condition, long-term treatment is likely to be needed in EOSS. ${ }^{1}$ There have been numerous randomized trials to determine the long-term effectiveness of antipsychotic agents in adults with schizophrenia, most of which have described few, if any, differences in outcomes between these medications (reviewed by Johnsen \& Jørgensen). ${ }^{4}$ To our knowledge, there are no long-term, double-blind, prospective studies that have examined the effectiveness and safety of different antipsychotic agents for up to one year in juveniles. We describe the results of maintenance treatment in youths who participated in the acute 
TEOSS trial and were maintained on their original randomized treatment as maintenance therapy.

Our approach to these analyses was mainly descriptive. We anticipated that the benefits observed during acute treatment would persist and that the three medications would demonstrate different tolerability profiles, with olanzapine and risperidone being associated with greater weight gain and metabolic changes, risperidone with hyperprolactinemia, and molindone with akathisia.

\section{METHOD}

\section{Study Design}

The TEOSS study methods have been described in detail in prior publications. ${ }^{3,5}$ In this report we focus on the 44-week, double-blind extension phase that followed the 8-week, double-blind acute trial. The primary results of the acute study have been described previously. ${ }^{3}$

During maintenance, youths continued to receive the same antipsychotic drug that they were administered during the acute phase. Treatment response and side effects were assessed every 4 weeks. Patients could be seen more frequently if clinically indicated.

All study procedures were reviewed and approved by the institutional review boards at each of the 4 study sites. Participant safety was also monitored by the National Institute of Mental Health (NIMH) Data and Safety Monitoring Board. All participants and their guardians provided written informed assent and consent, respectively.

For the sake of clarity, interventions prior to receiving therapy in the acute study will be denoted as occurring at the baseline time point. Weeks 8-52 specifically refer to time points in this maintenance study. In other words, week eight is the start of the maintenance study.

\section{Participants}

Youths, ages 8 to 19 years, who met diagnostic criteria for schizophrenia, schizophreniform disorder, or schizoaffective disorder were enrolled in the acute 8-week trial. Diagnostic methods, inclusion/exclusion criteria, and baseline characteristics of the sample are described elsewhere.2, 3, 5 After acute treatment, participants who: 1) were adequately tolerating their initially assigned treatment; and 2) had an adequate response to treatment (defined by a $20 \%$ reduction in baseline Positive and Negative Syndrome Scale (PANSS)6 score plus a Clinical Global Impression-Improvement (CGI-I)7 score of 1 or 2, indicative of being "very much improved" or "much improved") were eligible to continue on maintenance therapy. In addition, seven patients who did not meet the acute phase responder criteria but who had improved sufficiently were allowed to continue into maintenance therapy because it was felt to be in the best clinical interest of the patients.

\section{Pharmacological Treatments}

Subjects were treated with flexible doses of molindone (10-140 mg/day), olanzapine (2.5-20 $\mathrm{mg} /$ day), or risperidone ( $0.5-6 \mathrm{mg} / \mathrm{day})$. Dose increases were based on clinical need. All youths who were assigned to treatment with molindone also received $0.5 \mathrm{mg}$ twice daily of benztropine in order to reduce the risk of extrapyramidal symptoms (EPS). All other patients received matching placebo. Flexible dosing within these dose ranges was continued during the maintenance phase.

Adjunctive psychotropics permitted included: benztropine or trihexyphenidyl (for EPS); propranolol (for akathisia); lorazepam or clonazepam (for agitation and insomnia); fluoxetine, 
sertraline, or citalopram (serotonin selective reuptake inhibitors, "SSRIs," for depression), and valproate or lithium ("mood stabilizers" for manic symptoms). Diphenydramine could be prescribed for upper respiratory symptoms or insomnia. It is classified as a "psychotropic" for this manuscript due to the fact that diphenhydramie can also reduce EPS (although it was not used primarily for that purpose in this trial). Trazodone could be prescribed for insomnia. These adjunctive agents could be discontinued if they were no longer clinically indicated.

\section{Efficacy Measures and Outcomes}

The PANSS (a 30-item, clinician-rated scale used for the comprehensive assessment of psychotic symptoms), the Brief Psychiatric Rating Scale for Children (BPRS-C)8 (an assessment for the severity of 18 common psychiatric and behavioral problems with subscales for positive and negative symptoms, depression, and mania), and the Clinical Global Impression-Severity (CGI-S)7 (measurement of illness severity) were administered at all study visits. The Child and Adolescent Functional Assessment Scale (CAFAS) 9 was administered at baseline and weeks 8, 24 and 52/end of study (EOS).

\section{Adverse Event and Safety Monitoring}

In addition to open-ended inquiry about the presence of adverse events, the Monitoring of Side Effects Scale (MOSES) 10 was also used to ascertain adverse events. Events of moderate severity or higher which were not judged to be related to the underlying psychopathology were recorded as adverse events. EPS were monitored using the Neurological Rating Scale (NRS), 11 the Barnes Akathisia Scale (BAS), 12 and the Abnormal Involuntary Movement Scale (AIMS). 7 Vital signs, height, weight, and body mass index (BMI), 13 were also assessed. With the exception of the AIMS (obtained at baseline and weeks 8, 24, 36, and 52/end of study), other measures were obtained at each visit.

Between February 2002 and December 2005, subjects who gained more than 25\% of their pretreatment body weight were reviewed by the investigators in order to determine whether study withdrawal was appropriate. In December 2005, all subjects who had gained more than $7 \%$ of their body weight were reviewed in order to determine whether the apparent benefits experienced by the participants justified the associated risks. If a participant's BMI was $\geq 30$ $\mathrm{kg} / \mathrm{m}^{2}$ and the participant had gained more than $30 \%$ of their body weight since their pretreatment assessment, treatment discontinuation was required regardless of symptom response. Electrocardiograms (ECGs) were obtained at baseline and weeks 8, 24, 36, 52/ end of study. ECGs were read by a pediatric specialist in order to monitor for possible $\mathrm{QT}_{\mathrm{c}}$ prolongation.

Routine fasting blood and urine chemistries were obtained at baseline and weeks 8, 24, 36, and 52/end of study. Prolactin and thyroid-stimulating hormone (TSH) were measured at baseline, week 8 , and week 52/end of study. Prolactin was also measured at weeks 24 and 36 . Also, urine toxicology screens and pregnancy tests were collected at weeks 8 and 52/end of study. If a female subject was receiving adjunctive valproate or lithium, additional pregnancy tests were done at weeks 24 and 36 .

\section{Statistical Analyses}

Continuous variables were analyzed via F-tests in a one-way analysis of variance (ANOVA) with last observation carried forward change from baseline as outcome. To investigate possible violations of distributional assumptions due to small treatment group sizes, a nonparametric Kruskal-Wallis (three groups) or Wilcoxon test (2 groups) was also performed as a sensitivity analysis for each continuous outcome. Total scores for patients with missing items in their PANSS or CAFAS evaluations were weighted to reflect the number of items completed. Data for glucose, cholesterol, triglycerides, HDL, and LDL were included only if the patient was fasting at the time of laboratory data collection. 
Categorical outcomes, including proportions of patients taking concomitant medications or experiencing adverse events, were compared across treatment groups using exact chi-square tests. Concomitant medications were categorized post-hoc by the lead investigator using the text descriptions given in the database. Adverse events were categorized using their assigned MOSES category, except those with MOSES category "Other", for which a new adverse event category was created post-hoc by the lead investigator. To assess the presence of tardive dyskinesia, patient records were reviewed post-hoc by the investigators for any patient having 1) two or more items on the AIMS with scores 2 or higher, 2) one or more items on the AIMS with score 3 or higher, or 3 ) a score on any of the neurological scales of the MOSES of 3 or higher.

Time to discontinuation was estimated via Kaplan Meier survival curves and compared among treatment groups using a log-rank test. Time to discontinuation for each patient was computed by subtracting the dates of the baseline and termination study visits.

Due to the exploratory and descriptive purposes of this paper, no adjustments for multiple comparisons were made. All analyses were conducted using SAS software (Cary, NC) version 9.

\section{RESULTS}

\section{Subjects and Baseline Characteristics}

Figure 1 shows subject disposition during TEOSS. Of the 116 patients initially receiving therapy, 54 (47\%) entered this continuation study. Demographic and clinical characteristics of the entire TEOSS patient population at baseline are described elsewhere. ${ }^{2}$

Patients who only participated in the acute trial showed few statistically significant differences in demographics or baseline clinical features when compared to patients who continued with maintenance therapy. Youth taking olanzapine that entered this extension study had significantly lower mean baseline PANSS positive symptom scores than those who only participated in the acute trial $(23.6 \pm 5.1,28.1 \pm 5.8$, respectively; $\mathrm{p}=0.028)$. Conversely, for patients treated with risperidone, subjects receiving long-term treatment had higher mean baseline PANSS positive scores than those who only participated in the acute trial $(28.7 \pm 6.4$, 24.7 $\pm 5.9 ; \mathrm{p}=0.041$ ).

Additionally, patients receiving long-term olanzapine treatment had lower systolic blood pressure $(108.9 \pm 7.5,118.5 \pm 13.3, \mathrm{p}=0.023)$ than the olanzapine patients who only participated in the acute trial. Patients treated with long-term molindone had lower baseline sitting pulse $(73.5 \pm 9.5,80.4 \pm 11.8, \mathrm{p}=0.047$, ANOVA F-test, $\mathrm{p}=0.073$, Wilcoxon text $)$, which became non-significant in the sensitivity analysis.

Demographic information and week 8 and baseline clinical characteristics of the 54 participants are given in Table 1 and Table 2. The three treatment groups differed at with respect to gender: $12(92 \%)$ of patients receiving olanzapine were male, compared to $16(76 \%)$ and $9(45 \%)$ of the patients receiving risperidone and molindone, respectively $(\mathrm{p}=0.009)$. Patients treated with olanzapine had higher week 8 aspartate aminotransferase $(\mathrm{p}=0.004)$, higher baseline sitting pulse ( $\mathrm{p}=0.033$ ), and higher fasting triglycerides than the other treatment groups, although this later finding became non-significant in the nonparametric sensitivity analysis for $(\mathrm{p}=0.041$, ANOVA F-test; $p=0.068$, Wilcoxon test). Patients treated with risperidone had higher week 8 prolactin ( $\mathrm{p}<0.0001)$. Patients treated with molindone had lower week 8 systolic blood pressure $(\mathrm{p}=0.030)$. Also, patients treated with monlindone were prescribed significantly more concomitant benztropine (beyond the prophylactic benztropine prescribed to all patients treated with molindone) at week $8(\mathrm{p}=0.016)$. Otherwise, demographic and clinical characteristics at 
week 8 did not differ between the three medication treatment groups. Treatment groups differed at baseline with respect to BPRS-C $(\mathrm{p}=0.032)$ and use of concomitant benzodiazepines $(\mathrm{p}=0.037)$, although these differences dissipated by the start of maintenance therapy.

\section{Treatment Discontinuation}

Only 14 (26\%) of the 54 youths completed the 44-week extension trial (Figure 1). The most common reasons for study discontinuation were adverse events $(\mathrm{N}=15)$, inadequate efficacy $(\mathrm{N}=14)$, and non-adherence with study-related procedures $(\mathrm{N}=8)$. Average time to discontinuation during the maintenance study was $29.9 \pm 14.2$ weeks among patients receiving molindone, $23.0 \pm 16.6$ weeks among patients receiving olanzapine, and $25.3 \pm 15.2$ among patients receiving risperidone. Patient time to discontinuation is displayed graphically in Figure 2. While there were no statistically significant treatment group differences in length of maintenance study participation ( $\mathrm{p}=0.467)$, the rate of patient discontinuation in the group treated with molindone was consistently slower than that of the group treated with olanzapine.

\section{Antipsychotic Pharmacotherapy}

The mean doses of study medication at week 8 and week 52/end of study, respectively, were $66.5 \pm 38.7 \mathrm{mg} /$ day and $76.5 \pm 47.2 \mathrm{mg} /$ day for patients receiving molindone, $10.2 \pm 5.1 \mathrm{mg} / \mathrm{day}$ and $9.6 \pm 5.4 \mathrm{mg} /$ day for patients receiving olanzapine, and $3.3 \pm 1.5 \mathrm{mg} /$ day and $3.9 \pm 1.4 \mathrm{mg} /$ day for patients receiving risperidone. No statistically significant differences in antipsychotic dosage were observed during the maintenance phase in any treatment group.

\section{Concomitant Pharmacotherapy}

Forty-nine (91\%) patients took some concomitant medication during the extension trial (Table 3); 42 (78\%) took a concomitant psychotropic medication. Patients taking molindone were significantly more likely to have taken a psychotropic concomitant medication during the longterm study $(\mathrm{p}=0.018)$, due to the fact that more molindone-treated patients were taking concomitant benztropine at week 8 . However, there was no difference among the treatment groups with respect to the number of patients who began additional adjunctive benztropine or any other concomitant medication during this long-term extension trial.

\section{Outcome Measures}

Table 4 shows mean change from week 8 and change from baseline in clinical outcome measures. Like the acute study, there were no statistically significant differences between treatment groups in any of the maintenance phase clinical outcomes. However, the change in CAFAS 8 Total Score during maintenance was notably higher (indicative of worse functioning) in the group treated with risperidone (molindone: mean change $=0.7 \pm 33.9$; olanzapine: mean change $=3.3 \pm 32.8$; risperidone: mean change $=29.4 \pm 50.9$ ) and was significantly different from zero $(\mathrm{p}=0.025)$.

PANSS total score, the a priori primary outcome measure, as well as all other clinical outcome measures, varied little over the course of maintenance therapy. Last observation carried forward mean change from week 8 in PANSS total score was no larger than seven points in either direction at any of the time points in any treatment group. Despite the lack of significant change in clinical outcomes during maintenance therapy, significant differences in clinical measures seen during the acute phase continued to be maintained over the course of the entire 52 week trial ( $\mathrm{p} \leq 0.05$ for all variables and treatment groups).

\section{Safety and Tolerability}

Adverse Events-There were no significant differences among the treatment groups in adverse events at the beginning of the long term extension. Twenty (37\%) patients were 
experiencing at least one adverse event at week 8 , but no more than $4(7 \%)$ of all patients experienced any one type of adverse event at that time point.

The most common reason for study discontinuation during maintenance was adverse events. Forty-five (83\%) of all patients experienced at least one adverse event during the long term extension (Table 5). Weight gain (39\% of all patients) and anxiety (26\% of all patients) were the most common adverse events reported, but the rates of these did not significantly differ across the treatment groups. Weight gain was associated with treatment discontinuation in 11 patients (Figure 1). All 11 patients who discontinued from TEOSS due to weight gain had increases in their BMI z-scores during both the acute and maintenance phases. Five of these patients had greater increases in their BMI z-scores during the acute phase, 3 had larger gains in their BMI z-score while in the maintenance phase, and 3 had equal increases in BMI z-score during both phases. Pacing or restlessness was significantly more common in patients receiving molindone $(\mathrm{p}=0.018$ ). One serious adverse event, acute suicidal ideation in a patient taking risperidone, was observed.

Neurological Side Effects-Despite the fact that youths taking molindone reported significantly more adverse events of pacing or restlessness, no between treatment group differences were seen in change in NRS total, BAS, and AIMS total neurological measures during the maintenance study or over the entire 52 week study period (Table 4). Of note, no patient had persistent abnormal involuntary movement suggestive of tardive dyskinesia.

Weight Gain-All treatment groups showed statistically significant within-group weight gain and BMI increases during maintenance (Table 4). These increases became less or nonsignificant for standardized z-score and percentile measures. In contrast to the antecedent 8 week acute trial, there were no significant differences between treatment groups in change in weight, weight z-score, BMI, BMI percentile, or BMI z-score during the maintenance phase. However, patients treated with olanzapine did maintain significant increases in weight $z$-score, BMI percentile, and BMI z-score over molindone patients during the entire 52 week study $(\mathrm{p}=0.030 ; \mathrm{p}=0.010 ; \mathrm{p}=0.039$ respectively).

Laboratory Parameters and Vital Signs-In contrast to the acute trial, patients in the long term extension generally showed no significant differences across treatment groups in change from week 8 in laboratory parameters or vital signs and significant treatment differences in total cholesterol, LDL, insulin, ALT, and AST seen in the acute study were not maintained over the 52 week trial (Table 6). Patients receiving risperidone again saw the largest change in prolactin levels versus the other treatment groups during maintenance, but in the opposite direction. In the long term extension, patients receiving risperidone showed an average decrease from baseline of $11.2 \pm 14.1 \mathrm{mcg} / \mathrm{L}$ ( $\mathrm{p}=0.004$ for test of change different than zero). Despite this decrease, patients receiving risperidone maintained higher prolactin levels than other treatment groups throughout the entire 52 week trial, although this finding became nonsignificant in the sensitivity analysis ( $\mathrm{p}=0.045$, ANOVA F-test, $\mathrm{p}=0.077$, Wilcoxon test). In contrast to the acute phase of the study, patients receiving molindone showed the highest numerical increases in total cholesterol, LDL cholesterol, insulin, alanine aminotransferase, and aspartate aminotransferase. Patients treated with olanzapine showed a significant change from baseline in fasting glucose, though this became non-significant in the nonparametric sensitivity analysis ( $\mathrm{p}=0.011$, ANOVA F-test; $\mathrm{p}=0.125$, Wilcoxon test). Although patients treated with olanzapine had significantly greater increases in pulse and diastolic blood pressure during the antecedent acute trial ( $\mathrm{p}=0.002$, and $\mathrm{p}=0.031$, respectively), in comparison to those treated with risperidone or molindone, only patients treated with molindone showed a significant increase from baseline in systolic blood pressure $(\mathrm{p}=0.013)$ during maintenance therapy. Further, acute differences in pulse and diastolic blood pressure were not maintained over the entire 52 week study. 


\section{DISCUSSION}

Overall, $12 \%$ of youth enrolled in TEOSS completed 52 weeks on their originally randomized treatment. There were no differences in time to discontinuation or symptom reduction between the three study agents. Across acute and maintenance data, discontinuation rates due to either inadequate response and/or adverse events are high. In comparison to adult schizophrenia trials, these findings suggest that youth are less likely to maintain the same treatment over one year. ${ }^{14-17}$ In addition, symptom response tended to plateau during maintenance therapy, such that most youth did not make significant improvements beyond what they had achieved at 8 weeks of acute treatment.

Adverse events during maintenance treatment were common. Of the 15 subjects who discontinued their study medication due to side effects, 11 stopped because of weight gain. Each medication arm was associated with a significant increase in weight. None of the treatment groups significantly differed in regards to weight gain or other metabolic parameters. Akathisia was reported significantly more often by youth taking molindone. Elevated prolactin concentrations noted in subjects on risperidone at the end of the acute trial reduced over maintenance treatment, but remained elevated at end of study participation.

The lack of significant group metabolic differences during maintenance therapy is perhaps surprising. Acute TEOSS data found that olanzapine and risperidone were associated with significant weight gain, and olanzapine was associated with lipid and liver function changes.

${ }^{3}$ However, those analyses included subjects that discontinued after participation in the acute study due to metabolic side effects, whereas these maintenance data include only those that tolerated acute treatment and continued in this trial. Therefore, there is a selection bias towards patients that did not develop clinically significant adverse events during acute therapy.

Our maintenance therapy data are limited primarily by sample size and hence statistical power. Although TEOSS is the largest publicly funded treatment study of EOSS conducted to date, the sample size for maintenance data provides minimal power to assess for treatment arm differences. Thus, failure to reject a null hypothesis of no difference among treatments should not be taken as evidence of no difference. Our inclusion and exclusion criteria may have also limited generalizability. It should also be noted that it is possible that other available medications that were not studied in TEOSS would have been more effective and/or tolerable. Despite these limitations, a key strength of this work is its extended period of blinded treatment. Another strength of this study is that it demonstrates the pivotal role that medication tolerability has in the treatment of these vulnerable youth. Such an observation has real world significance.

The maintenance data presented in this paper suggest that these three agents generally maintain symptom reductions achieved in acute responders with EOSS. However, all three drugs have significant side effects that affect tolerability as shown by the fact that very few patients completed one year of treatment.

These data support the need for longer term comparative treatment trials for this patient population. Short-term placebo-controlled studies are limited in clinical utility if early responses are not maintained with continued therapy. Ultimately, more effective and safer treatments are needed for EOSS.

\section{Acknowledgments}

This study was supported by NIMH grants MH-61355 (Dr. Findling) to Case Western Reserve University, MH-61464 (Dr. McClellan) to the University of Washington, MH-62726 (Dr. Frazier) to Harvard Medical School, and MH-61528 (Dr. Sikich) to the University of North Carolina. Drs. Sikich (K23 MH- 01802) and Hlastala (K23 MH70570) were also supported by NIH career development awards. The research was conducted in NIH supported Clinical Research 
Centers at Seattle Children's Hospital, University of Washington (M01-RR-00037) and at the University of North Carolina at Chapel Hill (M01-RR00046 and U54RR024383).

The authors would like to thank Brieana M Rowles, MA for her technical assistance in drafting this manuscript. Ms. Rowles is with Case Western Reserve University.

\section{REFERENCES}

1. American Academy of Child and Adolescent Psychiatry. Practice parameter for the assessment and treatment of children and adolescents with schizophrenia. J Am Acad Child Adolesc Psychiatry 2001;40(7 Suppl):4S-23S.

2. Frazier JA, McClellan J, Findling RL, et al. Treatment of Early-Onset Schizophrenia Spectrum disorders (TEOSS): demographic and clinical characteristics. J Am Acad Child Adolesc Psychiatry 2007;46(8):979-988.

3. Sikich L, Frazier JA, McClellan J, et al. Double-blind comparison of first- and second-generation antipsychotics in early-onset schizophrenia and schizoaffective disorder: findings from the Treatment of Early-Onset Schizophrenia Spectrum Disorders (TEOSS) study. Am J Psychiatry 2008;165(11): 1420-1431.

4. Johnsen E, Jørgensen HA. Effectiveness of second generation antipsychotics: A systematic review of randomized trials. BMC Psychiatry 2008;8:31. [PubMed: 18439263]

5. McClellan J, Sikich L, Findling RL, et al. Treatment of Early-Onset Schizophrenia Spectrum Disorders (TEOSS): rationale, design, and methods. J Am Acad Child Adolesc Psychiatry 2007;46(8):969-978.

6. Kay SR, Fiszbein A, Opler LA. The positive and negative syndrome scale (PANSS) for schizophrenia. Schizophr Bull 1987;13(2):262-276.

7. National Institute of Mental Health. Special feature: rating scales and assessment instruments for use in pediatric psychopharmacology research. Psychopharmacol Bull 1985;21(4):765-770.

8. Overall JE, Pfefferbaum B. The brief psychiatric rating scale for children. Psychopharmacol Bull 1982;18(2):10-16. [PubMed: 7111598]

9. Hodges, K. Child and Adolescent Functional Assessment Scale. Ypsilanti: Department of Psychology, Eastern Michigan University; 1995.

10. Kalachnik, JE. Standardized monitoring for psychopharmacologic medication side effects. Manual for the Monitoring of Side Effects Scale (MOSES). Columbia, SC: University of South Carolina, School of Medicine; 2001.

11. Simpson GM, Angus JWS. A rating scale for extrapyramidal effects. Acta Psychiatr Scand 1970;212:111-199.

12. Barnes TR. A rating scale for drug-induced akathisia. Br J Psychiatry 1989;154:672-676. [PubMed: 2574607]

13. Centers for Disease Control and Prevention (CDC), National Center for Health Statistics (NCHS). National Health and Nutrition Examination Survey Data. Hyattsville, MD: U.S. Department of Health and Human Services, Centers for Disease Control and Prevention; 2000. http://www.cdc.gov/growthcharts/

14. Lieberman JA, Stroup TS, McEvoy JP, et al. Clinical Antipsychotic Trials of Intervention Effectiveness (CATIE) Investigators. Effectiveness of antipsychotic drugs in patients with chronic schizophrenia. N Engl J Med 2005;353(12):1209-1223.

15. Jones PB, Barnes TR, Davies L, et al. Randomized controlled trial of the effect on Quality of Life of second- vs first-generation antipsychotic drugs in schizophrenia: Cost Utility of the Latest Antipsychotic Drugs in Schizophrenia Study (CUtLASS 1). Arch Gen Psychiatry 2006;63(10):1079_ 1087.

16. Kahn RS, Fleischhacker WW, Boter H, et al. EUFEST study group. Effectiveness of antipsychotic drugs in first-episode schizophrenia and schizophreniform disorder: an open randomised clinical trial. Lancet 2008;371(9618):1085-1097.

17. McEvoy JP, Lieberman JA, Perkins DO, et al. Efficacy and tolerability of olanzapine, quetiapine, and risperidone in the treatment of early psychosis: a randomized, double-blind 52-week comparison. Am J Psychiatry 2007;164(7):1050-1060. 


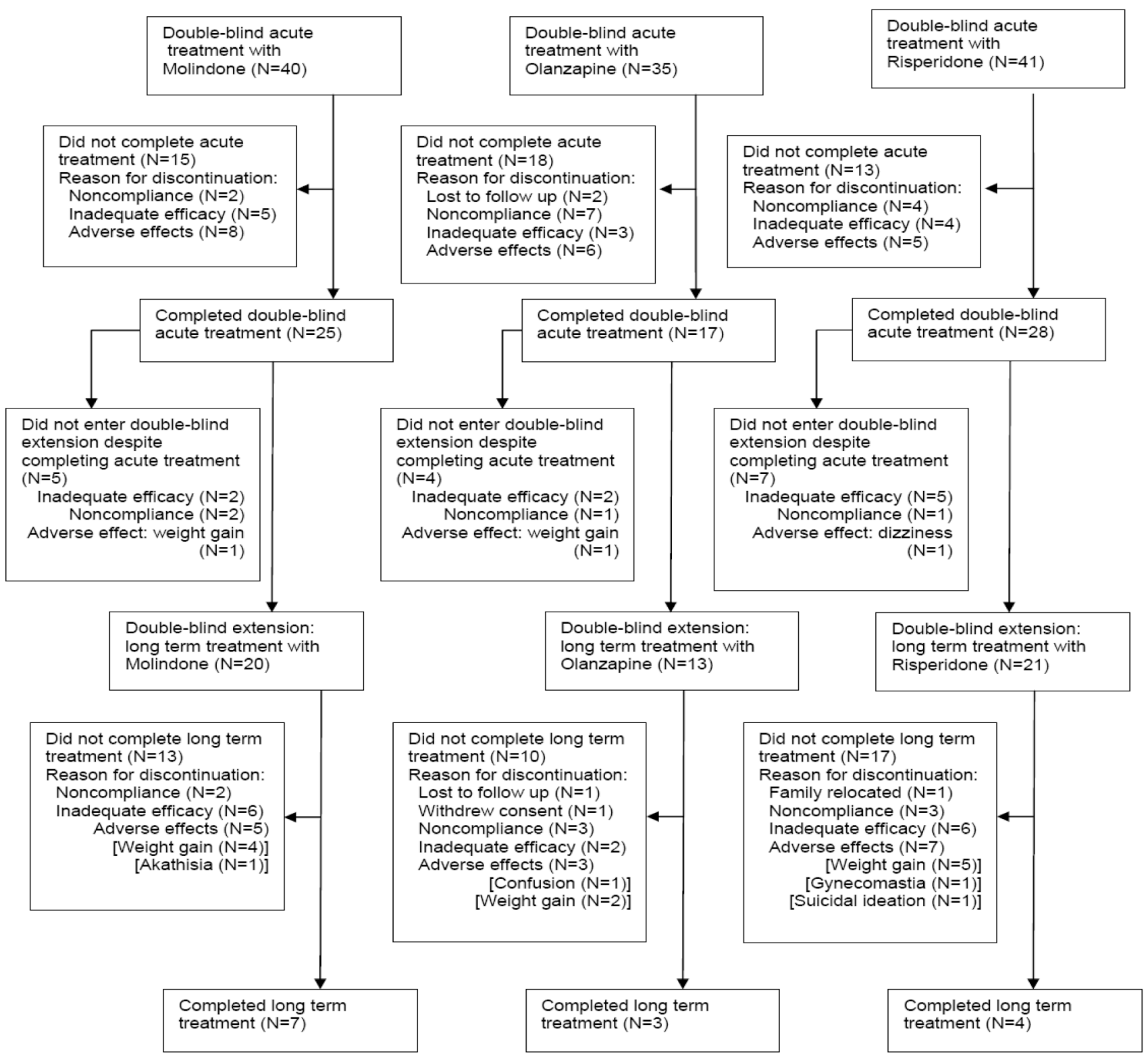

Figure 1.

Participant Disposition 


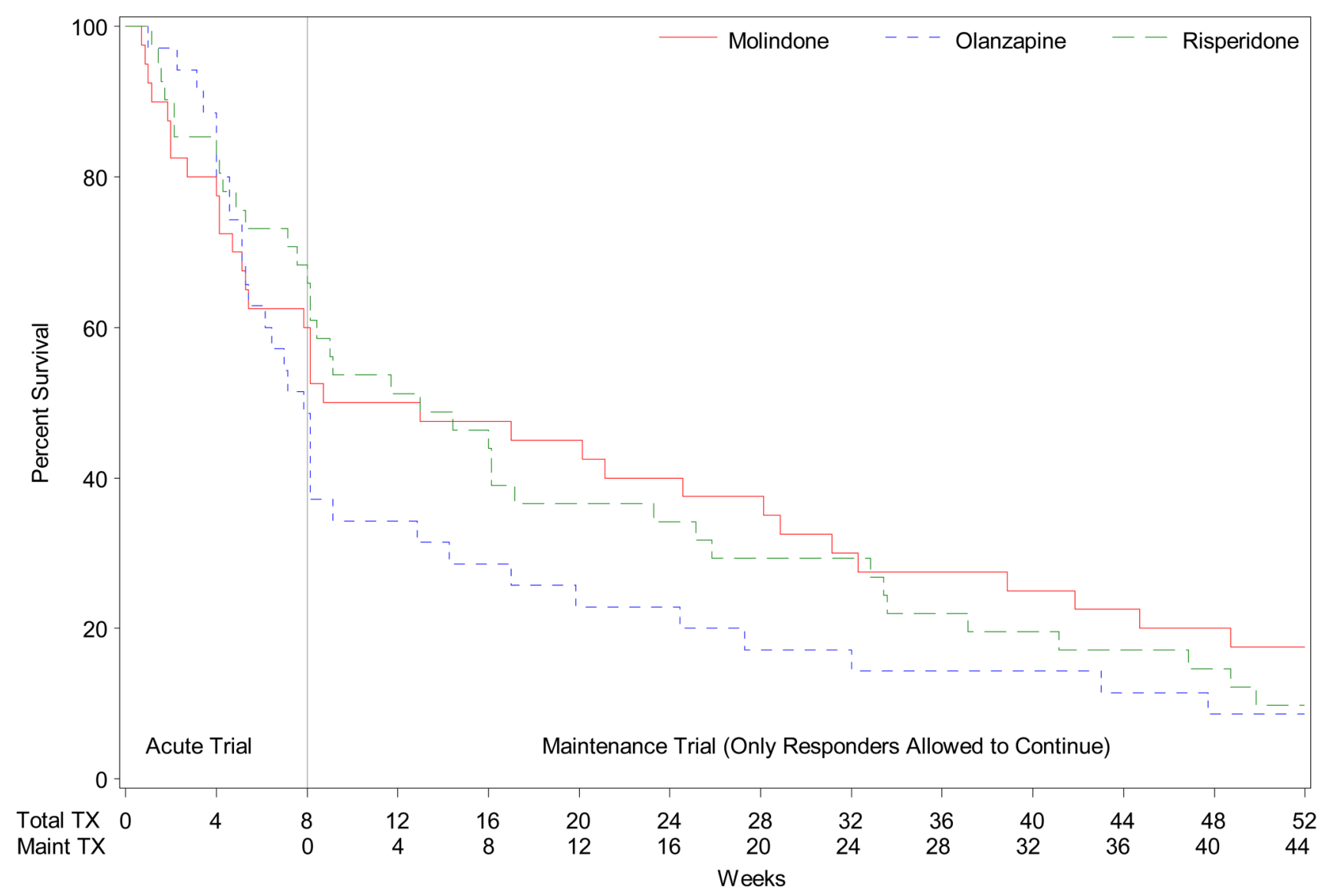

Figure 2.

Treatment discontinuation over time in the 52-week combined acute and maintenance studies 


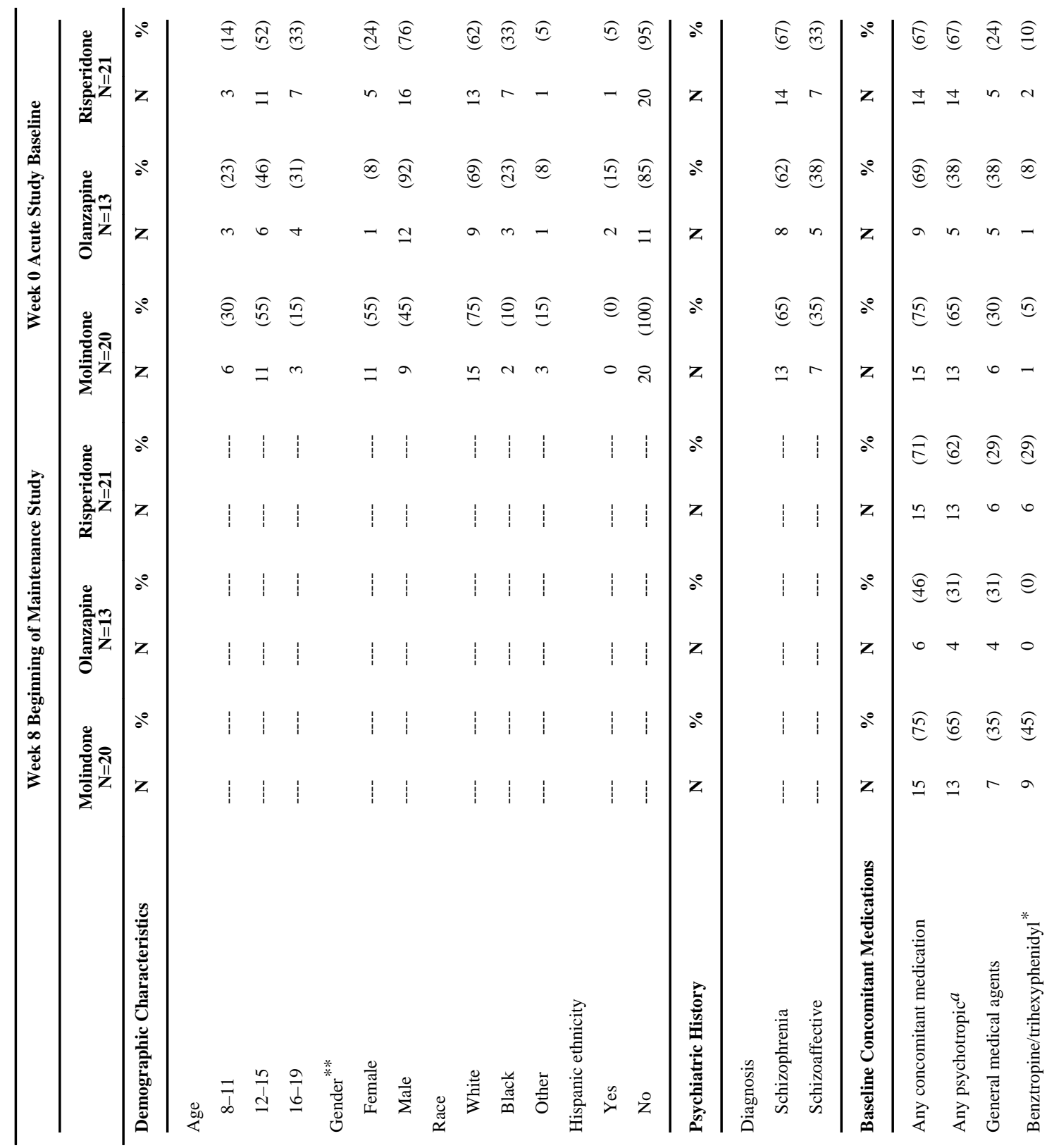




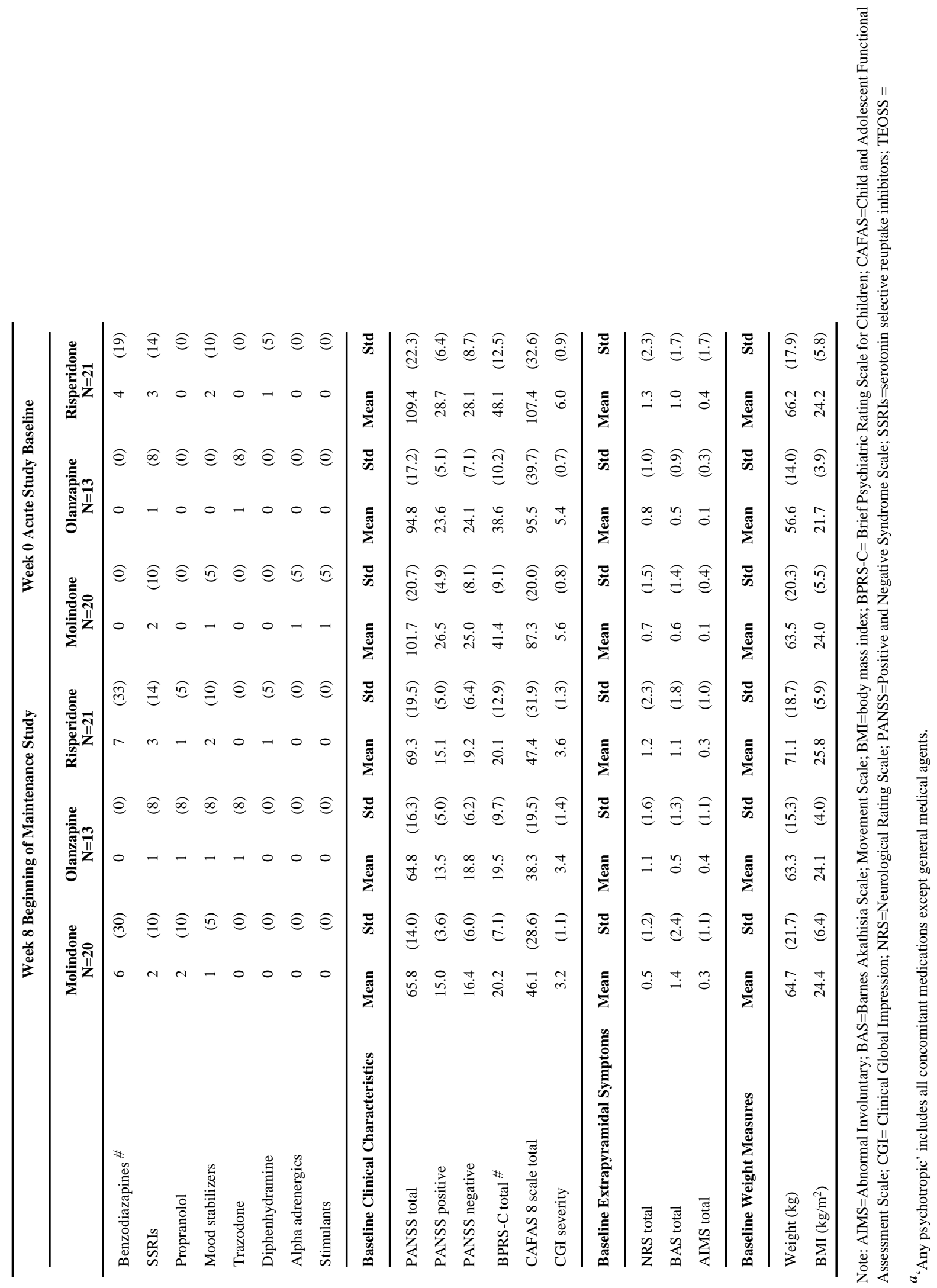




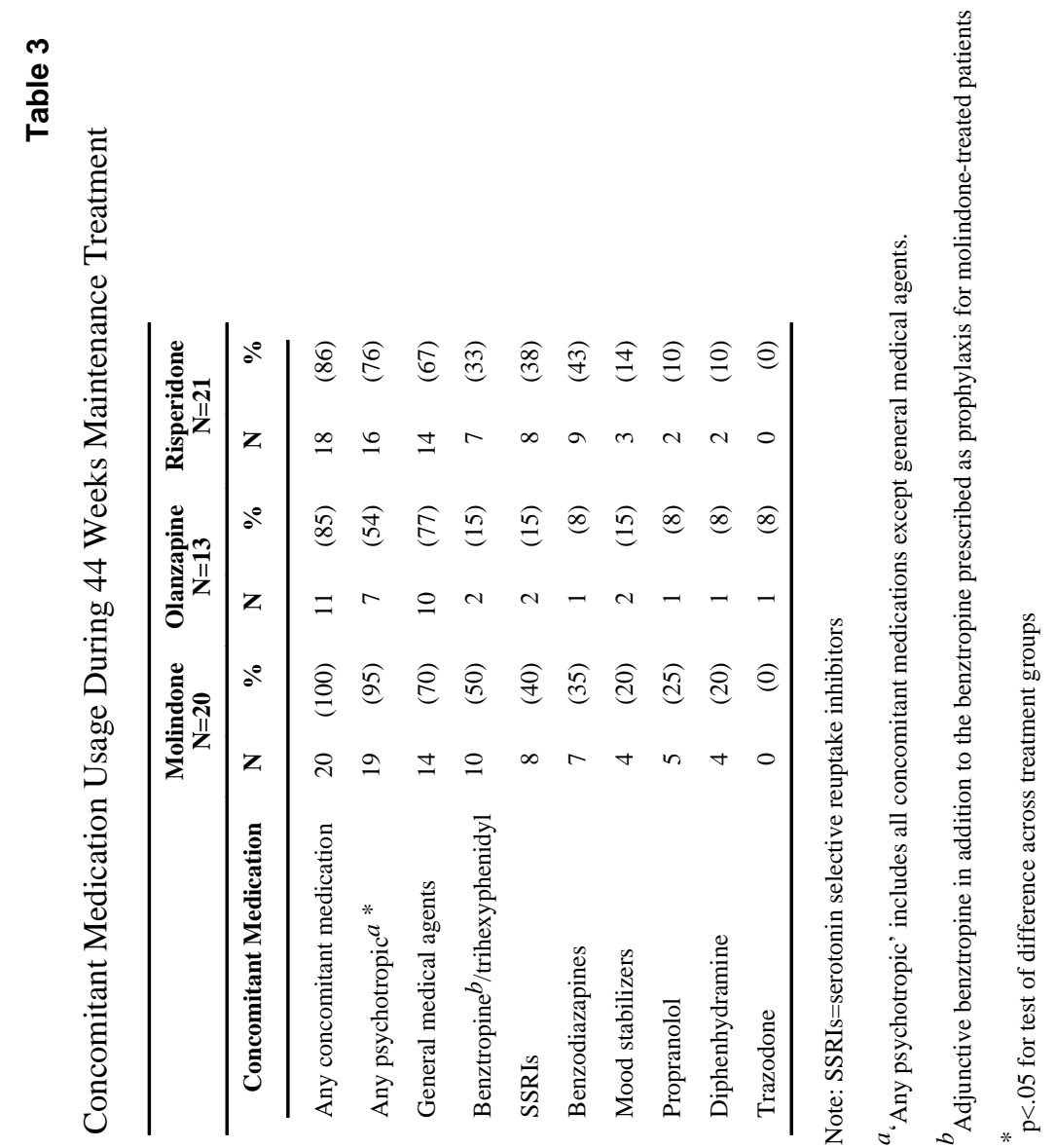




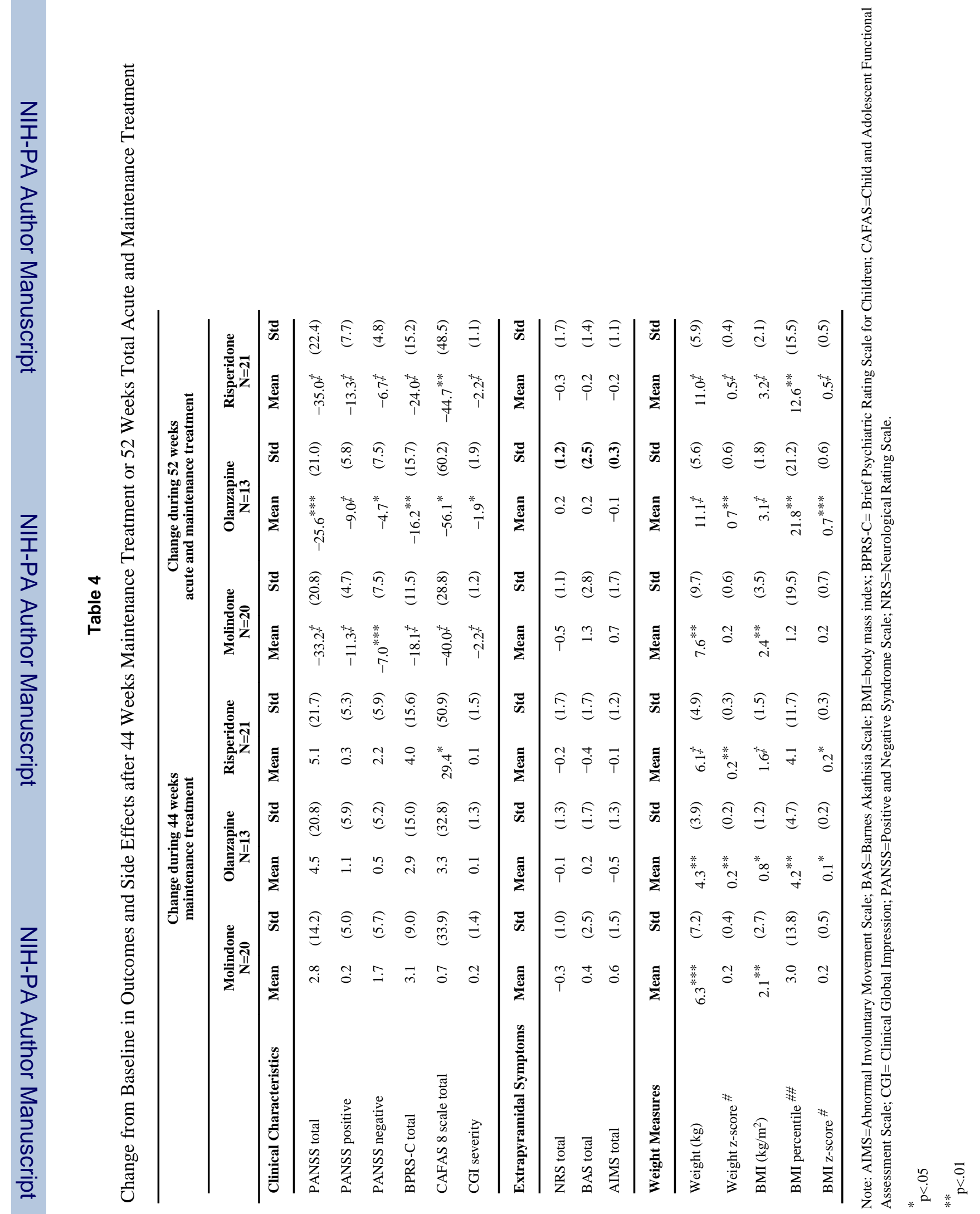

J Am Acad Child Adolesc Psychiatry. Author manuscript; available in PMC 2011 June 1. 
